\title{
Recent Developments of Domain-wall/Overlap Fermions for Lattice QCD
}

\author{
Ting-Wai Chiu ${ }^{\mathrm{a} *}$ \\ ${ }^{a}$ Physics Department, National Taiwan University, Taipei, Taiwan 106, Taiwan.
}

I review the lattice formulations of vector-like gauge theories (e.g. QCD) with domain-wall/overlap fermions, and discuss how to optimize the chiral symmetry for any finite $N_{s}$ (sites in the fifth dimension). In this formulation, quark propagators in gauge background can be computed efficiently through the effective $4 \mathrm{D}$ lattice Dirac operator.

\section{Introduction}

The basic idea of domain-wall fermions (DWF) $[1,2]$ is to use an infinite set of compled Dirac fermion fields $\left\{\psi_{s}(x), s \in(-\infty, \infty)\right\}$ with masses behaving like a step function $m(s)=$ $m \theta(s)$ such that Weyl fermion states can arise as zeromodes bound to the mass defect at $s=0$. However, if one uses a compact set of masses, then the boundary conditions of the mass (step) function must lead to the occurrence of both lefthanded and right-handed chiral fermion fields, i.e., a vector-like theory.

Exact chiral symmetry on the lattice was pioneered by Kaplan [3] with his proposal of domainwall fermions on the 5-dimensional lattice, in which the fifth dimension (internal flavor space) is discretized with $N_{s}$ sites (flavors) and lattice spacing $a_{5}$. Although the initial motivation was to provide a nonperturbative formulation of chiral gauge theories, the idea turns out to be natural and well-defined for vector-like gauge theories (e.g. QCD), with quark fields constructed from the boundary modes with open boundary conditions $[4,5]$. Soon after Kaplan proposed DWF for chiral gauge theories, Narayanan and Neuberger [6] observed that the chiral determinant can be written as the inner-product ("overlap") of two fermionic many body states of two bilinear Hamiltonians. For vector gauge theories like QCD, the fermion determinant is the product of a complex conjugate pair of chiral determinants, thus it is gauge invariant, real, non-negative, and the

\footnotetext{
*This work was supported in part by National Science Council, R.O.C. under the grant numbers NSC91-2112M002-025 and NSC92-2112-M002-023.
}

corresponding lattice Dirac operator for massless quarks (i.e., the overlap Dirac operator [7]) can be represented by a finite matrix of fixed shape regardless of the topology of the background gauge field, without undesired doubling or any finetuning. Mathematically, the overlap Dirac operator is exactly equal to the effective $4 \mathrm{D}$ lattice Dirac operator (for internal fermions dressed with pseudofermions) of DWF (with $m_{q}=0$ ) in the limit $N_{s} \rightarrow \infty$ followed by $a_{5} \rightarrow 0$,

$D=m_{0}\left(1+\gamma_{5} \frac{H_{w}}{\sqrt{H_{w}^{2}}}\right), \quad H_{w}=\gamma_{5} D_{w}$

where $D_{w}$ is the standard Wilson Dirac operator plus a negative parameter $-m_{0}\left(0<m_{0}<2\right)$.

For lattice QCD with DWF, in practice, one can only use a finite number $\left(N_{s}\right)$ of lattice Dirac fermion fields to set up the domain wall, thus the chiral symmetry of the quark fields (in the massless limit) is broken. Also, the discretization in the fifth dimension introduces $a_{5}$ into the theory. Presumably, only in the limit $N_{s} \rightarrow \infty$ and $a_{5} \rightarrow 0$, the correct effective $4 \mathrm{D}$ theory with exact chiral symmetry can be recovered. Since $N_{s}$ and $a_{5}$ are independent parameters, it might happen that even in the limit $N_{s} \rightarrow \infty$, the massless quark propagator is chirally symmetric but still depends on $a_{5}$, and similarly for the fermion determinant. Thus, even if $a_{5}$ is an irrelevant parameter (which decouples in the continuum limit), it is interesting to see whether $a_{5}$ can be eliminated completely at finite lattice spacing $a$, or whether it can be turned into a set of parameters ("variable spacings") such that the chiral symmetry can be preserved optimally. 
It turns out that for the conventional DWF with open boundary conditions [4], its quark propagator and effective 4D Dirac operator do not possess the optimal chiral symmetry for any finite $N_{s}$, and they depend on $a_{5}$ even at $N_{s}=\infty$, through the Hermitian operator $H$ (11) in the transfer matrix.

In this talk, I focus on the topics how to construct DWF action for lattice QCD [8-10] such that the quark propagator and the effective $4 \mathrm{D}$ lattice Dirac operator possess optimal chiral symmetry for any finite $N_{s}$ and gauge background. Due to the time constraint, I could not review recent numerical results on lattice QCD with domain-wall/overlap fermions.

\section{Lattice QCD with conventional DWF}

First, we examine the action ${ }^{2}$ of conventional DWF with open boundary conditions $[4,5]$

$$
\begin{aligned}
\mathcal{A}_{F}=\bar{\Psi} \mathcal{D}_{F} \Psi= & \sum_{s, s^{\prime}=1}^{N_{s}} \sum_{x, x^{\prime}} \bar{\psi}(x, s)\left[a_{5} D_{w}\left(x, x^{\prime}\right) \delta_{s, s^{\prime}}\right. \\
& \left.+\delta_{x, x^{\prime}} D_{5}\left(s, s^{\prime}\right)\right] \psi\left(x^{\prime}, s^{\prime}\right), \quad \text { (2) }
\end{aligned}
$$

where $D_{w}$ is the Wilson-Dirac operator minus a parameter $m_{0}\left(0<a_{5} m_{0}<2,0<m_{0}<2\right)$, and

$$
\begin{aligned}
D_{5}\left(s, s^{\prime}\right) & =\delta_{s, s^{\prime}}-P_{-} \delta_{s^{\prime}, s+1}-P_{+} \delta_{s^{\prime}, s-1}, \\
P_{ \pm} & =\frac{1}{2}\left(1 \pm \gamma_{5}\right),
\end{aligned}
$$

with boundary conditions:

$$
\begin{aligned}
P_{+} \psi(x, 0) & =-r m_{q} P_{+} \psi\left(x, N_{s}\right), \\
P_{-} \psi\left(x, N_{s}+1\right) & =-r m_{q} P_{-} \psi(x, 1), \\
r & =\left[m_{0}\left(2-a_{5} m_{0}\right)\right]^{-1} .
\end{aligned}
$$

Here $m_{q}$ is the bare quark mass, and the quark fields coupling to physical hadrons are constructed from the boundary modes:

$$
\begin{gathered}
q(x)=\sqrt{r}\left[P_{-} \psi(x, 1)+P_{+} \psi\left(x, N_{s}\right)\right] \\
\bar{q}(x)=\sqrt{r}\left[\bar{\psi}(x, 1) P_{+}+\bar{\psi}\left(x, N_{s}\right) P_{-}\right] .
\end{gathered}
$$

To regularize the fermion determinant, PauliVillars (pseudofermion) fields $\left\{\phi_{x s}, \bar{\phi}_{x s}\right\}$ carrying

\footnotetext{
${ }^{2}$ Here we suppress the lattice spacing $a$, and the Dirac, flavor, and color indices.
}

the same (flavor, color, Dirac) indices of $\{\psi, \bar{\psi}\}$ but obeying the Bose statistics are introduced [11, 6]. The action of pseudofermions $\mathcal{A}_{P F}$ is exactly the same as (2) except replacing $\{\psi, \bar{\psi}\}$ by $\{\phi, \bar{\phi}\}$, with boundary conditions $\left(r m_{q}=1\right)$ :

$$
\begin{aligned}
P_{+} \phi(x, 0) & =-P_{+} \phi\left(x, N_{s}\right), \\
P_{-} \phi\left(x, N_{s} \text { । 1 }\right) & =-P_{-} \phi(x, 1) .
\end{aligned}
$$

Thus the total action of the system is

$$
\mathcal{A}=\mathcal{A}_{G}+\mathcal{A}_{F}+\mathcal{A}_{P F}
$$

where $\mathcal{A}_{G}$ is the gluon action.

Then the fermion determinant, the effective $4 \mathrm{D}$ lattice Dirac operator [12], and the quark propagator [13] in background gauge field can be obtained by integrating over all heavy fermion fields and pseudofermion fields. In general, the generating functional for $n$-point Green's function of quark fields $q$ and $\bar{q}$ can be derived as [10]

$Z[J, \bar{J}]=\frac{\int[d U] e^{-\mathcal{A}_{G}[U]} \operatorname{det} D\left(m_{q}\right) e^{\bar{J}\left(D_{c}+m_{q}\right)^{-1} J}}{\int[d U] e^{-\mathcal{A}_{C}[U]} \operatorname{det} D\left(m_{q}\right)}$

where $\bar{J}(x)$ and $J(x)$ are the Grassman sources of $q(x)$ and $\bar{q}(x)$ respectively, and

$$
\begin{aligned}
r D_{c} & =\frac{1+\gamma_{5} S(H)}{1-\gamma_{5} S(H)} \\
S(H) & =\frac{1-T^{N_{s}}}{1+T^{N_{s}}} \equiv a_{5} H R\left(a_{5}^{2} H^{2}\right) \\
T & =\frac{1-a_{5} H}{1+a_{5} H} \\
H & =\gamma_{5} \frac{D_{w}}{2+a_{5} D_{w}} \\
D\left(m_{q}\right) & =\left(D_{c}+m_{q}\right)\left(1+r D_{c}\right)^{-1} \\
& =\left[1+r m_{q}+\left(1-r m_{q}\right) \gamma_{5} S(H)\right] / 2 r \\
D\left(m_{q}\right)^{-1} & =\left(1-r m_{q}\right)\left(D_{c}+m_{q}\right)^{-1}+r
\end{aligned}
$$

The most remarkable feature of DWF is the emergence of the quark propagator $\left(D_{c}+m_{q}\right)^{-1}$ which preserves all vital symmetries of its counterpart $\left[\gamma_{\mu}\left(\partial_{\mu}+i g A_{\mu}\right)+m_{q}\right]^{-1}$ in continuum, while the sea quark propagator $D\left(m_{q}\right)^{-1}$ for internal quark loops is equal to $\left(D_{c}+m_{q}\right)^{-1}$ times a constant factor $\left(1-\mathrm{ram}_{q}\right)$ plus a constant ra, as shown in Eq. (13).

In the limit $N_{s} \rightarrow \infty, S(H) \rightarrow \frac{H}{\sqrt{H^{2}}}=\operatorname{sgn}(H)$, then the quark propagator $\left(D_{c}+m_{q}\right)^{-1}$ in the 
massless limit $\left(m_{q} \rightarrow 0\right)$ is chirally symmetric. Consequently, the effective 4D lattice Dirac operator for massless internal quark loops, $D(0)$ $D_{c}\left(1+r D_{c}\right)^{-1}=\left[1+\gamma_{5} S(H)\right] / 2 r \equiv D$, satisfies the Ginsparg-Wilson relation [14]

$D \gamma_{5}+\gamma_{5} D=2 r D \gamma_{5} D$.

Further, taking $a_{5} \rightarrow 0$, then $S(H) \rightarrow \operatorname{sgn}\left(H_{w}\right)$, and $D$ is exactly equal to the overlap Dirac operator (1). (But $a_{5}$ must be nonzero at finite $N_{s}$ ).

Several remarks are as follows.

(i) The axial anomaly is recovered through the Chern-Simmons current $[15,4]$.

(ii) In the limit $N_{s} \rightarrow \infty$, the non-singlet flavor symmetry $S U_{L}\left(N_{f}\right) \times S U_{R}\left(N_{f}\right)$ of $N_{f}$ massless quarks is exact at finite lattice spacing [4].

(iii) The effective 4D lattice Dirac operator $D\left(m_{q}\right)$ is exponentially-local for sufficiently smooth background gauge fields [16-18].

(iv) Any quark observable in QCD can be obtained from $Z[J, \bar{J}]$ by differentiation, and it possesses the discrete symmetries $(C, T, P)$ of its counterpart in continuum.

(a) Quark propagator

$$
\begin{gathered}
\langle q(x) \bar{q}(y)\rangle=-\left.\frac{\delta^{2} Z[J, \bar{J}]}{\delta \bar{J}(x) \delta J(y)}\right|_{J=\bar{J}=0} \\
=\frac{\int[d U] e^{-\mathcal{A}_{C}} \prod_{f} \operatorname{det} D\left(m_{f}\right)\left(D_{c}+m_{q}\right)_{x, y}^{-1}}{\int[d U] e^{-\mathcal{A}_{C}} \prod_{f} \operatorname{det} D\left(m_{f}\right)}
\end{gathered}
$$

(b) Current-current correlator

$$
\begin{aligned}
& \left\langle\bar{d}(x) \gamma_{4} P_{-} s(x) \bar{s}(0) \gamma_{4} P_{-} d(0)\right\rangle \\
= & \left.\frac{\delta}{\delta J_{d}(x)} \gamma_{4} P_{-} \frac{\delta}{\delta \bar{J}_{s}(x)} \frac{\delta}{\delta J_{s}(0)} \gamma_{4} P_{-} \frac{\delta}{\delta \bar{J}_{d}(0)} Z[J, \bar{J}]\right|_{n} \\
= & \frac{\int[d U] e^{-\mathcal{A}_{C}} \prod_{f} \operatorname{det} D\left(m_{f}\right) O_{K}(x)}{\int[d U] e^{-\mathcal{A}_{C}} \prod_{f} \operatorname{det} D\left(m_{f}\right)}
\end{aligned}
$$

where

$O_{K}(x)=-\operatorname{tr}\left[\left(D_{c}+m_{d}\right)_{0, x}^{-1} \gamma_{4} P_{-}\left(D_{c}+m_{s}\right)_{x, 0}^{-1} \gamma_{4} P_{-}\right]$.

Note that the V-A structure of the left-handed quark currents is preserved exactly.

(v) If one uses the effective 4D lattice Dirac operator $D\left(m_{q}\right)$ (or any GW Dirac operator) to define the quark action, then subtleties might emerge. (Recall that $\mathrm{CP}$ in chiral gauge theories with GW fermion is broken by $O(a)$ effect [19]).
Consider the massless $\left(m_{q}=0\right)$ case,

$$
\begin{aligned}
& \mathcal{A}_{F}=\sum_{x, y} \bar{q}(x) D_{x, y} q(y) \equiv \bar{q} D q \\
= & \sum_{x, y}\left[\bar{q}(x) P_{1} D_{x, y} \hat{P}_{-} q(y)+\bar{q}(x) P_{-} D_{x, y} \hat{P}_{+} q(y)\right]
\end{aligned}
$$

where $\hat{P}_{ \pm}=\frac{1}{2}\left[1 \pm \gamma_{5}(1-2 r D)\right]$ and $P_{ \pm}=(1 \pm$ $\left.\gamma_{5}\right) / 2$ are the chiral projectors for the quark fields $q$ and $\bar{q}$ respectively. Then the left-handed quark current does not manifest the V-A structure, e.g. $\bar{d}(x) \gamma_{\mu} \hat{P}_{-} s(x) \neq \bar{d}(x) P_{+} \gamma_{\mu} s(x)$. Consequently, the effective weak Hamiltonian constructed from these left-handed quark currents would break the $S U_{L}(2)$ gauge symmetry by $O(a)$ effect [20].

Note that this problem cannot be resolved by re-defining the quark field as $\hat{q}=(1-r D) q=$ $\left(1+r D_{c}\right)^{-1} q$, since $\mathcal{A}_{F}=\bar{q} D q=\bar{q} D_{c} \hat{q}$, where $D_{c}$ becomes nonlocal as $N_{s} \rightarrow \infty$.

Thus, for any Ginsparg-Wilson fermion action, it is impossible to define local quark fields with exponentially-local kernel such that the correlation function of quark fields in gauge background can be expressed as

$$
\langle q(x) \bar{q}(y)\rangle=\left(D_{c}+m_{q}\right)_{x, y}^{-1} .
$$

Even though $\left(D_{c}+m_{q}\right)^{-1}$ can be computed via $D\left(m_{q}\right)^{-1}$, conceptually, the valence quark fields have to be defined in terms of the boundary modes of domain-wall fermions.

Nevertheless, the conventional DWF has its own deficiencies:

(a) For any finite $N_{s}, S(H)$ is not the optimal rational approximation for $\operatorname{sgn}(H)[8]$, thus the chiral symmetry is not preserved optimally.

(b) Even for $N_{s}=\infty, S(H)=H / \sqrt{H^{2}}$ still depends on $a_{5}$ through $H=\gamma_{5} D_{w}\left(2+a_{5} D_{w}\right)^{-1}$.

In the following, I discuss how to solve these two problems.

\section{Zolotarev optimal rational polynomial}

The deviation of $S(H)$ from $\operatorname{sgn}(H)$ can be measured in terms of

$$
\begin{aligned}
\sigma(S) & =\max _{\forall Y \neq 0}\left|\frac{Y^{\dagger}\{\operatorname{sgn}(H)-S(H)\} Y}{Y^{\dagger} Y}\right| \\
& \leq \max _{\{\eta\}}|\operatorname{sgn}(\eta)-S(\eta)|,
\end{aligned}
$$


where $\{\eta\}$ are eigenvalues of $a_{5} H$. Using the simple identity

$|\operatorname{sgn}(x)-S(x)|=\left|1-\sqrt{x^{2}} R\left(x^{2}\right)\right|$

where $S(x)=x R\left(x^{2}\right)$, we can rewrite (14) as

$\sigma(S) \leq \max _{\left\{\eta^{2}\right\}}\left|1-\sqrt{\eta^{2}} R\left(\eta^{2}\right)\right|$

where $\left\{\eta^{2}\right\}$ are eigenvalues of $a_{5}^{2} H^{2}$. Clearly the problem of finding the optimal rational approximation $S_{\text {opt }}(x)$ of $\operatorname{sgn}(x)$ with $x \in$ $\left[-x_{\max },-x_{\min }\right] \cup\left[x_{\min }, x_{\max }\right]$ is equivalent Lo finding the optimal rational approximation $R_{\text {opt }}\left(x^{2}\right)$ of $\left(x^{2}\right)^{-1 / 2}$ with $x^{2} \in\left[x_{\min }^{2}, x_{\max }^{2}\right]$.

According to de la Vallée-Poussin's theorem and Chebycheff's theorem[21], the necessary and sufficient condition for an irreducible rational polynomial (where $m \geq n, p_{i}, q_{i}>0$ )

$r^{(n, m)}(x)=\frac{p_{n} x^{n}+p_{n-1} x^{n-1}+\cdots+p_{0}}{q_{m} x^{m}+q_{m-1} x^{m-1}+\cdots+q_{0}}$

to be the optimal rational polynomial of the inverse square root function $x^{-1 / 2}, 0<x_{\text {min }} \leq$ $x \leq x_{\max }$ is that $\delta(x) \equiv 1-\sqrt{x} r^{(n, m)}(x)$ has $n+m+2$ alternate change of sign in the interval $\left[x_{\min }, x_{\max }\right]$, and attains its maxima and minima (all with equal magnitude), say,

$\delta(x)=-\Delta,+\Delta, \cdots,(-1)^{n+m+2} \Delta$

at consecutive points $\left(x_{i}, i=1, \cdots, n+m+2\right)$

$x_{m i n}=x_{1}<x_{2}<\cdots<x_{n \mid-m+2}=x_{\max }$.

In other words, if $r^{(n, m)}$ satisfies the above condition, then its error

$\sigma\left(r^{(n, m)}\right)=\max _{x \in\left[x_{r n i n}, x_{n n, m, x}\right]}\left|1-\sqrt{x} r^{(n, m)}(x)\right|$

is the minimum among all irreducible rational polynomials of degree $(n, m)$.

It has been shown [8] that $R\left(x^{2}\right)$ (9) of the conventional DWF (i.e., the polar approximation) does not satisfy above criterion, thus is not the optimal rational approximation for $\left(x^{2}\right)^{-1 / 2}$.

The optimal rational approximation for inverse square root function $\left(x^{2}\right)^{-1 / 2}, x^{2} \in[1, b]$ was first obtained by Zolotarev in 1877 , using Jacobian clliptic functions. Explicitly,

$R_{Z}^{(n, n)}\left(x^{2}\right)=d_{0} \prod_{l=1}^{n} \frac{1+x^{2} / c_{2 l}}{1+x^{2} / c_{2 l-1}}$, and

$R_{Z}^{(n-1, n)}\left(x^{2}\right)-d_{0}^{\prime} \frac{\prod_{l=1}^{n-1}\left(1+x^{2} / c_{2 l}^{\prime}\right)}{\prod_{l=1}^{n}\left(1+x^{2} / c_{2 l-1}^{\prime}\right)}$,

where the coefficients $d_{0}, d_{0}^{\prime}, c_{l}$ and $c_{l}^{\prime}$ are expressed in terms of elliptic functions [21] with arguments depending only on $n$ and $b$. A detailed discussion of Zolotarev's result can be found in Akhiezer's two books [21]. (see also a recent discussion [22] in the context of lattice QCD.)

The first application of Zolotarev optimal rational polynomial in latticc QCD was to approximate [23] $\left(H_{w}^{2}\right)^{-1 / 2}$ in the overlap Dirac operator (1), which improves the (optimal) rational approximation [24] via the Remes algorithm ${ }^{3}$.

For lattice QCD with DWF at finite $N_{s}$, the optimization problem is how to construct a DWF action such that the operator $S(H)(9)$ is replaced with

$S_{\text {opt }}(H)= \begin{cases}h R_{Z}^{(n, n)}\left(h^{2}\right), & N_{s}=2 n+1, \\ h R_{Z}^{(n-1, n)}\left(h^{2}\right), & N_{s}=2 n,\end{cases}$

where $h=H / \lambda_{\min }$, and $b=\lambda_{\max }^{2} / \lambda_{\min }^{2}\left(\lambda_{\min }\right.$ and $\lambda_{\max }$ are the minimum and the maximum of the eigenvalues of $|H|)$. However, this could not be solved for the conventional DWF, due to the functional form of $H=\gamma_{5} D_{w}\left(2+a_{5} D_{w}\right)^{-1}$.

\section{Borici's variant of DWF}

For the effective 4D Dirac operator $D\left(m_{q}\right)(12)$ in the fermion determinant, it turned out to be possible to modify the conventional DWF action such that its $S(H)$ is replaced by $S\left(H_{w}\right)$.

The prescription [25] is to replace $\delta_{x, x^{\prime}} D_{5}\left(s, s^{\prime}\right)$ in (2) with

$\delta_{x, x^{\prime}} \delta_{s, s^{\prime}}+\left(a_{5} D_{w}-1\right)_{x, x^{\prime}}\left(P_{-} \delta_{s^{\prime}, s+1}+P_{+} \delta_{s^{\prime}, s-1}\right)$.

The boundary conditions (3)-(4) and quark fields (6)-(7) are the same except replacing $r$ (5) with $r=1 /\left(2 m_{0}\right)$. After introducing pseudofermion

\footnotetext{
${ }^{3}$ The Remes algorithm is a numerical (iterative) scheme to implement the criterion of de la Vallée-Poussin's theorem and Chebycheff's theorem to obtain optimal rational approximation of any continuous function $f(x)$. However, in the case $\left(x^{2}\right)^{-1 / 2}$, Remes algorithm must give less precise numerical coefficients than those computed directly from Zolotarev's exact solution.
} 
fields $\left(r m_{q}=1\right)$, the fermion determinant can be evaluated as

$$
\begin{gathered}
\operatorname{det}\left(\mathcal{D}_{F} \mathcal{D}_{P F}^{-1}\right) \equiv \operatorname{det} D \\
=\operatorname{det}\left\{m_{q}+\left(m_{0}-m_{q} / 2\right)\left[1+\gamma_{5} S\left(H_{w}\right)\right]\right\} .
\end{gathered}
$$

In the limit $N_{s} \rightarrow \infty, S\left(H_{w}\right) \rightarrow H_{w}\left(H_{w}^{2}\right)^{-1 / 2}$, then the fermion determinant (19) is independent of $a_{5}$.

However, unlike the conventional DWF, its quark propagator in gauge background [26] is abnormal,

$\langle q(x) \bar{q}(y)\rangle \neq\left(D_{c}+m_{q}\right)_{x, y}^{-1}$,

thus its quark propagator as well as other quark observables do not manifest the discrete symmetries of their counterparts in continuum, similar to the problem encountered in the GinspargWilson fermion.

\section{Optimal domain-wall fermion (ODWF)}

The problem (20) can be solved $[10]$ by appending two new boundary layers at $s=0$ and $s=N_{s}+1$, both with the constraint $a_{5}=0$, and re-defining the quark fields with these new boundary modes.

Further, one can turn $a_{5}$ at each layer into a parameter $\omega_{s}$. Then this set of parameters $\left\{\omega_{s}\right\}$ can be constructed such that $S\left(H_{w}\right)$ is equal to $S_{\text {opt }}\left(H_{m}\right)$, the optimal rational approximation for $\operatorname{sgn}\left(H_{w}\right)[8]$.

The action of optimal domain-wall fermion [10] can be written as

$$
\begin{aligned}
& \mathcal{A}_{F}=\bar{\Psi} \mathcal{D}_{o d w} \Psi=\sum_{s, s^{\prime}=0}^{N_{s}+1} \sum_{x, x^{\prime}} \bar{\psi}_{x s}\left\{\left(\omega_{s} D_{w}+1\right)_{x x^{\prime}} \delta_{s s^{\prime}}\right. \\
& \left.+\left(\omega_{s} D_{w}-1\right)_{x x^{\prime}}\left(P_{+} \delta_{s^{\prime}, s-1}+P_{-} \delta_{s^{\prime}, s+1}\right)\right\} \psi_{x^{\prime} s^{\prime}}(21)
\end{aligned}
$$

with boundary conditions

$$
\begin{aligned}
P_{+} \psi(x,-1) & =-r m_{q} P_{+} \psi\left(x, N_{s}+1\right), \\
P_{-} \psi\left(x, N_{s}+2\right) & =-r m_{q} P_{-} \psi(x, 0), r=\frac{1}{2 m_{0}},
\end{aligned}
$$

where $\omega_{0}-\omega_{N_{s}+1}=0$, and other $\left\{\omega_{s}\right\}$ are fixed as follows. The quark fields are constructed as

$$
\begin{aligned}
& q(x)=\sqrt{r}\left[P_{-} \psi(x, 0)+P_{+} \psi\left(x, N_{s}+1\right)\right] \\
& \bar{q}(x)=\sqrt{r}\left[\bar{\psi}(x, 0) P_{+}+\bar{\psi}\left(x, N_{s}+1\right) P_{-}\right] .
\end{aligned}
$$

Then the quark propagator in gauge background can be derived as $[10]$

$$
\langle q(x) \bar{q}(y)\rangle=\left(D_{c}+m_{q}\right)_{x, y}^{-1}
$$

where

$$
\begin{aligned}
D_{c} & =2 m_{0} \frac{1+\gamma_{5} S_{o}\left(H_{w}\right)}{1-\gamma_{5} S_{o}\left(H_{w}\right)} \\
S_{o}\left(H_{w}\right) & =\frac{1-\prod_{s=1}^{N_{s}} T_{s}}{1+\prod_{s=1}^{N_{s}} T_{s}}, T_{s}=\frac{1-\omega_{s} H_{w}}{1+\omega_{s} H_{w}}
\end{aligned}
$$

Now requiring $S_{o}\left(H_{w}\right)$ equal to the optimal rational approximation of $\operatorname{sgn}\left(H_{w}\right)$ amounts to solving the parameters $\left\{\omega_{s}\right\}$ from the equation

$S_{o}\left(H_{w}\right)= \begin{cases}h_{w} R_{Z}^{(n, n)}\left(h_{w}^{2}\right), & N_{s}=2 n+1, \\ h_{w} R_{Z}^{(n-1, n)}\left(h_{w}^{2}\right), & N_{s}=2 n .\end{cases}$

This is equivalent to solving the roots $\left(u_{s}=\right.$ $\omega_{s}^{-2}, s=1, \cdots, N_{s}$ ) from the nonlinear equations

$1-\sqrt{u} R_{Z}^{(n, n)}(u)=0, \quad N_{s}=2 n+1$,

$1-\sqrt{u} R_{Z}^{(n-1, n)}(u)=0, \quad N_{s}=2 n$.

The exact solution of (25) has been obtained in [8], which gives

$\omega_{s}=\frac{1}{\lambda_{\min }} \sqrt{1-\kappa^{\prime 2} \mathrm{sn}^{2}\left(v_{s} ; \kappa^{\prime}\right)}, s=1, \cdots, N_{s}$

Here $\operatorname{sn}\left(v_{s} ; \kappa^{\prime}\right)$ is the Jacobian elliptic function with modulus $\kappa^{\prime}=\sqrt{1-\lambda_{\min }^{2} / \lambda_{\max }^{2}}$, and argument $v_{s}$

$v_{s}=(-1)^{s-1} M \mathrm{sn}^{-1}\left(\sqrt{\frac{1+3 \lambda}{(1+\lambda)^{3}}} ; \lambda^{\prime}\right)+\left[\frac{s}{2}\right] \frac{2 K^{\prime}}{N_{s}}$

where

$$
\begin{aligned}
M & =\prod_{l=1}^{\left[\frac{N_{s}}{2}\right]} \frac{\operatorname{sn}^{2}\left(\frac{(2 l-1) K^{\prime}}{N_{s}} ; \kappa^{\prime}\right)}{\operatorname{sn}^{2}\left(\frac{2 l K^{\prime}}{N_{s}} ; \kappa^{\prime}\right)}, \\
\lambda & =\prod_{l=1}^{N_{*}} \frac{\Theta^{2}\left(\frac{2 l K^{\prime}}{N_{s}} ; \kappa^{\prime}\right)}{\Theta^{2}\left(\frac{(2 l-1) K^{\prime}}{N_{s}} ; \kappa^{\prime}\right)}, \lambda^{\prime}=\sqrt{1-\lambda^{2}},
\end{aligned}
$$

$K^{\prime}$ is the complete elliptic integral of the first kind with modulus $\kappa^{\prime}$, and $\Theta$ is the elliptic theta function. From (26), one has $\lambda_{\max }^{-1} \leq \omega_{s} \leq \lambda_{\min }^{-1}$, since $\operatorname{sn}^{2}(;) \leq 1$. Note that $\lambda_{\min }\left(\lambda_{\max }\right)$ can be 
fixed to be the greatest lower bound (least upper bound) of the eigenvalues of $\left|H_{w}\right|$ for the set of gauge configurations under investigation. Clearly the $O D W F$ action (21) is always ultralocal, no matter how one fixes $\lambda_{\min }$ and $\lambda_{\max }$.

It should be pointed out that the new boundary layers at $s=0$ and $s=N_{s}+1$ with $\omega_{0}=$ $\omega_{N_{s}+1}=0$ were first introduced in [10], several months after the weights (26) had been obtained in [8]. The purpose of these two new boundary layers is to render the quark propagator satisfying (22) such that any quark observable manifests the discrete symmetries of its counterpart in continuum. Since $T_{0}=T_{N_{\mathrm{s}}+1}=1$, the transfer matrix as well as $S_{o}\left(H_{w}\right)$ are not affected by these two new boundary layers.

After introducing the pseudofermion fields $\left(m_{q}=2 m_{0}\right)$, the generating function for $n$-point function of $q$ and $\bar{q}$ can be derived as [10],

$Z[J, \bar{J}]=\frac{\int[d U] e^{-\mathcal{A}_{G}[U]} \operatorname{det} D\left(m_{q}\right) e^{\bar{J}\left(D_{c}+m_{q}\right)^{-1} J}}{\int[d U] e^{-\overline{\mathcal{A}}_{G}[U]} \operatorname{det} D\left(m_{q}\right)}$

where

$$
\begin{aligned}
D_{c} & =2 m_{0} \frac{1+\gamma_{5} S_{o p t}\left(H_{w}\right)}{1-\gamma_{5} S_{o p t}\left(H_{w}\right)} \\
D\left(m_{q}\right) & =m_{q}+\left(m_{0}-m_{q} / 2\right)\left[1+\gamma_{5} S_{o p t}\left(H_{w}\right)\right]
\end{aligned}
$$

It has been shown that $D\left(m_{q}\right)$ (for any $m_{q}$ and $N_{s}$ ) is exponentially-local for sufficiently smooth gauge backgrounds [9]. Note that for any finite $N_{s}, D(0)(28)$ is exactly equal to the overlap Dirac operator with $\left(H_{w}^{2}\right)^{-1 / 2}$ approximated by Zolotarev optimal rational polynomial, (16) $\left(N_{s}\right.$ = odd), or (17) $\left(N_{s}=\right.$ even $)$.

Since $S_{\text {opt }}\left(H_{w}\right)$ is the optimal rational approximation for $\operatorname{sgn}\left(H_{w}\right)$, its error is bounded uniformly over the entire range of $\left[1, \lambda_{\max }^{2} / \lambda_{\max }^{2}\right]$, and is the minimum among all rational polynomials of the same degree,

$\sigma\left(S_{\text {opt }}\right) \leq \frac{1-\lambda}{1+\lambda} \simeq A(b) e^{-c(b) N_{s}}, b=\lambda_{\max }^{2} / \lambda_{\min }^{2}$

where $\lambda$ is defined in (27), and

$$
\begin{aligned}
A(b) & \simeq 4.06(1) b^{-0.0091(1)} \ln (b)^{0.0042(3)} \\
c(b) & \simeq 4.27(45) \ln (b)^{-0.746(5)}
\end{aligned}
$$

which can be estimated by asymptotic expansion and numerical evaluation of $\lambda$.
Thus for any set of gauge configurations, one can determine what values of $N_{s}$ and $b$ (i.e., how many low-lying eigenmodes of $H_{w}^{2}$ should be projected out) are required to attain one's desired accuracy in preserving the chiral symmetry.

The quark propagator in gauge background

$$
\begin{gathered}
\langle q(x) \bar{q}(y)\rangle=\left(D_{c}+m_{q}\right)_{x, y}^{-1} \\
=\sum_{s, s^{\prime}}\left(P_{-} \delta_{s, 0}+P_{+} \delta_{s, N_{s}+1}\right) \mathcal{D}_{o d w}^{-1}\left(x, s ; y, s^{\prime}\right) \times \\
\left(P_{+} \delta_{s^{\prime}, 0}+P_{-} \delta_{s^{\prime}, N_{s}+1}\right)
\end{gathered}
$$

can be computed in two different ways:

(a) To solve the linear system of the 5D lattice Dirac operator

$\mathcal{D}_{\text {odw }}\left(x, s ; y, s^{\prime}\right) Y=\left(P_{+} \delta_{s, 0}+P_{-} \delta_{s, N_{s}+1}\right) \cdot \mathbf{I}$

(b) To solve the system of the effective 4D lattice Dirac operator

$$
\begin{aligned}
& D\left(m_{q}\right) D^{\dagger}\left(m_{q}\right) Y=\mathbf{I} \\
= & \left\{m_{q}^{2}+\left(m_{0}^{2}-m_{q}^{2} / 4\right)\left[2+\left(\gamma_{5} \pm 1\right) S_{o p t}\left(H_{w}\right)\right]\right\} Y
\end{aligned}
$$

with conjugate gradient, where the matrix-vector product $S_{o p t}\left(H_{w}\right) \cdot Y$ can be obtained by invoking another conjugate gradient (inner CG). It turns out that the best algorithm to compute $S_{o p t} \cdot Y$ is Neuberger's double pass algorithm [27].

At present, the scheme (b) seems to be much more efficient than the scheme (a), in terms of the CPU time and the memory space. Further, there are multi-shift $\mathrm{CG}$ algorithms available for the scheme (b) to compute quark propagators for a set of quark masses, but so far there is no multimass algorithms for the scheme (a). For a recent quenched QCD calculation via the scheme (b), see [28].

At this point, it is instructive to point out the remarkable features of Neuberger's double pass:

(i) the memory storage for the conjugate gradient is constant ( 5 vectors), independent of the degree $n$ of the rational polynomial $R^{(n-1, n)}$.

(ii) the CPU time is almost independent of $n$, thus the matrix-vector product $R \cdot Y$ can be approximated to very high precision (at large $n$ ) without noticably extra costs.

(iii) there exists a threshold $n_{T}$ such that the double pass algorithm is faster than the single 
pass algorilhın for $n>n_{T}$ (where $n_{T} \simeq 12-25$ for most platforms).

Note that the last two properties have just becn unveiled recently [29]. Undoubtedly, Neuberger's double pass algorithm will become the standard for computing $R\left(H^{2}\right) \cdot Y$ in lattice QCD.

\section{Conclusions}

A new DWF action (21) for lattice QCD has been constructed such that the quark propagator and the effective 4D Dirac operator for internal quark loops have optimal chiral symmetry for any $N_{s}$ and gauge background. The quark fields are constructed from the new boundary modes at $s=0$ and $s=N_{s}+1$ with $\omega_{0}=\omega_{N_{s}+1}=0$ such that the quark propagator satisfics (22) and any quark observable manifests the discrete symmetries of its counterpart in continuum. The quark propagator in gauge background can be computed efficiently through the effective 4D lattice Dirac operator, and the dynamical quarks can be simulated similar to the Wilson fermion in 5D.

\section{Acknowledgments}

I am grateful to the Organizers of Lattice 2003 for invitation and kind hospitality. I thank many colleagues for discussions, in particular, David Adams, David Kaplan, Yoshio Kikukawa, Rajamani Narayanan, Herbert Neuberger and Steve Sharpe. I also thank my (former) students, Tung-Han Hsieh, Chao-Hsi Huang and TsungRen Huang, for collaborations on numerical simulations of lattice QCD with a Linux PC cluster (hep-lat/0208039, currently of 64 nodes).

\section{REFERENCES}

1. V. A. Rubakov and M. E. Shaposhnikov, Phys. Lett. B 125, 136 (1983).

2. C. G. Callan and J. A. Harvey, Nucl. Phys. B 250, 427 (1985).

3. D. B. Kaplan, Phys. Lett. B 288, 342 (1992); Nucl. Phys. Proc. Suppl. 30, 597 (1993).

4. Y. Shamir, Nucl. Phys. B 406, 90 (1993)

5. V. Furman and Y. Shamir, Nucl. Phys. B 439, 54 (1995)

6. R. Narayanan and H. Neuberger, Phys. Lett.
B 302, 62 (1993); Nucl. Phys. B 412, 574 (1994); Phys. Rev. Lett. 71, 3251 (1993); Nucl. Phys. B 443, 305 (1995)

7. H. Neuberger, Phys. Lett. B 417, 141 (1998)

8. T. W. Chiu, Phys. Rev. Lett. 90, 071601 (2003)

9. T. W. Chiu, Phys. Lett. B 552, 97 (2003)

10. T. W. Chiu, hep-lat/0303008.

11. S. A. Frolov and A. A. Slavnov, Phys. Lett. B 309, 344 (1993).

12. H. Neuberger, Phys. Rev. D 57, 5417 (1998)

13. Y. Kikukawa and T. Noguchi, heplat/9902022.

14. P. H. Ginsparg and K. G. Wilson, Phys. Rev. D 25, 2649 (1982)

15. M. F. Golterman, K. Jansen and D. B. Kaplan, Phys. Lett. B 301, 219 (1993)

16. P. Hernandez, K. Jansen and M. Luscher, Nucl. Phys. B 552, 363 (1999)

17. H. Neuberger, Phys. Rev. D 61, 085015 (2000)

18. Y. Kikukawa, Nucl. Phys. B 584, 511 (2000)

19. P. Hasenfratz, Nucl. Phys. Proc. Suppl. 106, 159 (2002)

20. K. Fujikawa, M. Ishibashi and H. Suzuki, JHEP 0204, 046 (2002)

21. N. I. Akhiezer, Theory of approximation (Dover, New York, 1992); Elements of the theory of elliptic functions, Translations of Mathematical Monographs, 79, (American Mathematical Society, Providence, RI. 1990).

22. T. W. Chiu, T. H. Hsieh, C. H. Huang and T. R. Huang, Phys. Rev. D 66, 114502 (2002)

23. J. van den Eshof, A. Frommer, T. Lippert, K. Schilling and H. A. van der Vorst, Nucl. Phys. Proc. Suppl. 106, 1070 (2002)

24. R. G. Edwards, U. M. Heller and R. Narayanan, Nucl. Phys. B 540, 457 (1999)

25. A. Borici, Nucl. Phys. Proc. Suppl. 83, 771 (2000)

26. R. G. Edwards and U. M. Heller, Phys. Rev. D 63,094505 (2001)

27. H. Neuberger, Int. J. Mod. Phys. C 10, 1051 (1999)

28. T. W. Chiu and T. H. Hsieh, Nucl. Phys. B 673, 217 (2003) [hep-lat/0305016].

29. T. W. Chiu and T. H. Hsieh, hep-lat/0306025 